\title{
Multi-parameter measurements using optical fibre long period gratings for indoor air quality monitoring
}

\author{
Jiri Hromadka ${ }^{\mathrm{a}, \mathrm{b}}$, Sergiy Korposh ${ }^{\mathrm{a}, \mathrm{c}}$, Matthew C. Partridge ${ }^{\mathrm{c}}$, Stephen W. James ${ }^{\mathrm{c}, *}$, \\ Frank Davis ${ }^{\mathrm{d}}$, Derrick Crump ${ }^{\mathrm{e}}$, Ralph P. Tatam ${ }^{\mathrm{c}}$ \\ a Electrical Systems and Optics Research Division, Faculty of Engineering, University of Nottingham, University Park, Nottingham NG7 2RD, UK \\ ${ }^{\mathrm{b}}$ Institute for Environmental Studies, Faculty of Science, Charles University in Prague, CZ-128 01, Czechia \\ ' Engineering Photonics, Cranfield University, Cranfield, Bedfordshire MK43 OAL, UK \\ d Engineering Science, Cranfield University, Cranfield, Bedfordshire MK43 OAL, UK \\ e Institute for Environment, Health, Risk and Futures, Cranfield University, Cranfield, Bedfordshire MK43 OAL, UK
}

\section{A R T I C L E I N F O}

\section{Article history:}

Received 7 April 2016

Received in revised form 7 December 2016

Accepted 9 December 2016

Available online 11 December 2016

\section{Keywords:}

Long period grating (LPG)

Multiplexing

Phase match turning point (PMTP)

Indoor air quality (IAQ)

Relative humidity ( $\mathrm{RH})$

Volatile organic compounds (VOCs)

\begin{abstract}
A B S T R A C T
An array of three long period gratings (LPGs) fabricated in a single optical fibre and multiplexed in the wavelength domain was used to measure simultaneously temperature, relative humidity (RH) and volatile organic compounds (VOCs), which are key indoor air quality (IAQ) indicators. Each LPG sensor was designed with optimised response to a particular measurand. The first, with no surface modification, was used to measure temperature. The second, modified by a mesoporous coating of silica nanoparticles $\left(\mathrm{SiO}_{2} \mathrm{NPs}\right.$ ), was used to measure RH and the third, modified with a coating of $\mathrm{SiO}_{2} \mathrm{NPs}$ infused with a functional material, $p$-sulphanatocalix[8]arene (CA[8]), was employed to monitor VOC concentration. The LPGs were fabricated with periods such that they operated at or near the phase matching turning point. The sensors were calibrated in the laboratory and the simultaneous measurement of the key indoor air quality parameters was undertaken in laboratory and office environments. It was demonstrated successfully that the data produced by the LPG sensor array under real conditions was in a good agreement with that produced by commercially available sensors. The average differences between values obtained by the optical fibre sensor and standard temperature and $\mathrm{RH}$ sensors were better than $0.5{ }^{\circ} \mathrm{C}$ and $5 \%$ respectively. Further, the potential application of fibre optic sensors for VOC detection at high concentrations has been demonstrated.
\end{abstract}

(C) 2017 The Authors. Published by Elsevier B.V. This is an open access article under the CC BY license (http://creativecommons.org/licenses/by/4.0/).

\section{Introduction}

During the last decade, interest in air quality control has shifted from outdoor to indoor environments, reflecting the changes in lifestyle associated with increasing levels of urbanization [1]. Most people spend around $90 \%$ of their time indoors [2]. There is significant scientific evidence that indicates that inappropriate indoor air quality (IAQ) affects negatively human health and thus its monitoring plays a key role in IAQ control [3].

IAQ is influenced by a mixture of physical, chemical and biological factors, each with different sources and associated adverse health effects [3]. Temperature, relative humidity $(\mathrm{RH})$ and volatile organic compounds (VOCs) represent the key factors of interest

\footnotetext{
* Corresponding author.

E-mail address: s.w.james@cranfield.ac.uk (S.W. James).
}

[4]. Temperature extremes represent a serious risk for human health, where low temperature during winter months can cause cardiovascular diseases and death within susceptible groups such as elderly people [5]. Extreme heat can cause a range of adverse health effects with different severity, from heat rashes to heat stroke. Heat also negatively affects the respiratory and cardiovascular systems [6]. Low RH can cause irritation of the eyes and mucous membranes of the respiratory system, increase sensitivity to aerosol particles and facilitate the spread of airborne diseases [7]. On the other hand, high RH leads to higher occurrence of allergies that affect negatively respiratory systems, such as asthma, respiratory infections, coughs, wheeze and dyspnoea [8]. The recommended values of $\mathrm{RH}$ for human well-being and that minimize possible adverse health effects are in the range of $40-60 \%$ [7].

Temperature and RH are essential parameters in the assessment of the performance of a building, because of their influence on 
energy demand. The reduction of the energy needed to obtain the appropriate IAQ is important for both people's health and for utility costs. There is a need to develop technologies for future homes, which optimise performance against these criteria [9].

VOCs in the indoor environment comprise a broad mixture of chemicals that are present in household products and that leak from materials commonly present indoors (e.g. paints and furniture). VOCs are also product of combustion processes such as heating or smoking and their concentrations can increase by up to a 1000 times over short time periods. VOCs cause various short and longterm (delayed) adverse health effects [10], and VOCs are recognised as being one of the possible causes of sick building syndrome (SBS) [11].

While hundreds of VOCs are present in indoor air, the measurement and identification of every single one is difficult and expensive. The total VOCs (TVOCs) concentration is commonly measured and used as an IAQ parameter. There are several ways to measure the concentration of TVOCs, such as infrared detectors, passive badges, photo-ionization detectors and flame ionization detectors [10]. The most used approach, which gives the highest sensitivity, is active sorption/chemical analysis, which uses gas chromatography-mass spectrophotometry (GC-MS) for further analysis. The VOCs are measured individually and the TVOC is subsequently calculated [10]. Despite its sensitivity, the GC-MS approach is expensive, requires well-trained operators and a sample collection step that makes real time measurements impossible.

Fibre optic sensors can help to solve problems of IAQ monitoring, since they are inexpensive, small, lightweight, immune to electromagnetic interference and as such can be used in extreme conditions, enabling remote real time monitoring with no electrical power needed at the sensing point [12]. Fibre-optic sensing platforms based on long period gratings (LPGs) with functional coatings have been used to measure various measurands, including RH [13], ammonia [14], and VOCs [15]. In addition, when proteins, enzymes and antibodies are incorporated, then optical fibres can be used for biological response measurement [16]. Moreover, the potential use of fibre optic sensors for sick building syndrome (SBS) monitoring has been suggested [17].

Among the different types of fibre-optic sensors, those based on gratings, specifically long period gratings (LPGs), have been employed extensively for refractive index measurements [18] and for monitoring associated chemical processes [18], since they offer wavelength-encoded information, which overcomes the referencing issues associated with intensity based approaches. An LPG consists of a periodic perturbation of the refractive index of the fibre core, which couples the core mode to the co-propagating cladding modes of the fibre. This coupling is manifested in the transmission spectrum of the optical fibre as a series of resonance bands. Each resonance band corresponds to coupling to a different cladding mode and thus shows different sensitivity to environmental changes [19].

The coupling wavelength can be obtained from the following phase matching equation

$\lambda_{\mathrm{x}}=\left(\mathrm{n}_{\text {core }}-\mathrm{n}_{\mathrm{clad}(\mathrm{x})}\right) \Lambda$

Where $\lambda_{x}$ represents the wavelength at which light is coupled to the $\mathrm{LP}_{0 \mathrm{x}}$ cladding mode, $n_{\text {core }}$ is the effective refractive index of the mode propagating in the core of the fibre, $n_{\operatorname{clad}(x)}$ is the effective index of the $\mathrm{LP}_{0 \mathrm{x}}$ cladding mode and $\Lambda$ is the period of the LPG [19]. The central wavelength of the resonance band is sensitive to changes in environmental conditions such as strain, temperature, bend radius and refractive index of the surrounding conditions [19].
The thermal sensitivity of LPGs arises from a combination of the thermo-optic effect and the thermal expansion of the fibre. The sensitivity of the LPG can be increased by appropriate choice of the grating period and composition of the optical fibre [20]. The shift of the central wavelength of the resonance bands caused by a temperature change is generally linear from ambient temperatures to up to $150{ }^{\circ} \mathrm{C}[20]$.

An LPG's sensitivity to the refractive index of the medium surrounding the optical fibre is associated with the dependence of the effective refractive index of the cladding mode upon the surrounding environment [19]. Higher sensitivity and selectivity can be obtained by functional coating of the cladding. For instance, a $400 \mathrm{~nm}$ thick coating of silica sphere nanoparticles was shown to improve sensitivity of the LPG to changes in the refractive index of the surrounding medium [21].

It has been shown that the phase matching condition for each cladding mode contains a turning point and that the LPG exhibits the highest sensitivity when phase matching turning point is reached. This can be achieved by choosing an appropriate LPG period and coating thickness [15,22]. The cylindrical shape of the optical fibre represents a challenge for coating deposition. Three deposition techniques are widely used: the dip coating technique [23], the Langmuir-Blodgett technique [15,24] and the layer by layer technique, also known as electrostatic self-assembly [14,25-27].

The layer-by-layer technique is based on the deposition of oppositely charged materials that can be added to the fibre on the molecular level to build up a coating of the required thickness. The entire coating can be made from the sensitive material and then react with the compound of interest, leading to a change of refractive index of the coating. Another option is to infuse the functional compound into a porous coating, where again interaction with the measurand leads to a refractive index change [14].

One of the key advantages of fibre optic sensors is the ability to multiplex an array of sensors, sensitive to the same or to different parameters. This can be of significant benefit in real environments, where the influence of interfering factors such as temperature or relative humidity should be reduced. Simultaneous detection of several parameters at the same location using a single optical fibre offers additional information that allows correction for changes of the interfering parameters [19].

In this work, a sensor array consisting of 3 LPGs with different grating periods written in a single optical fibre was used for simultaneous measurements of temperature, relative humidity and concentration of VOCs. The LPGs have periods selected such that they all operate near the phase matching turning point and that differ by up to $1 \mu \mathrm{m}$ to facilitate wavelength division multiplexing. To the best of our knowledge, this is a first example of the multiplexing of the LPGs sensors operating at the phase matching turning point (PMTP). A mesoporous coating of silica nanospsheres was deposited onto LPG1, such that it was sensitive to RH. The surface of LPG2 was left unmodified and was used to measure temperature. A functional material, Calixarene, was infused into a mesoporous silica nanospshere coating deposited onto LPG3 to sensitise the LPG to VOCs [15,25]. Calixarene molecules contain of a number of phenol or resorcinol aromatic rings connected together to a larger ring and the molecule is shaped like a bowl [28]. The analyte of the interest reacts with calixarene and becomes temporarily entrapped via gas state complexion. As only weak interactions occur (no covalent bond is created) the analyte is liberated easily from the cavity, with the result that the sensor is reversible. The sensitivity of the reaction depends on the morphology and charge of the molecule of interest and for this reason semi-specific reactions to different VOCs have been reported $[15,25]$. The sensor array was tested in real environments to demonstrate the ability to measure key IAQ parameters. 


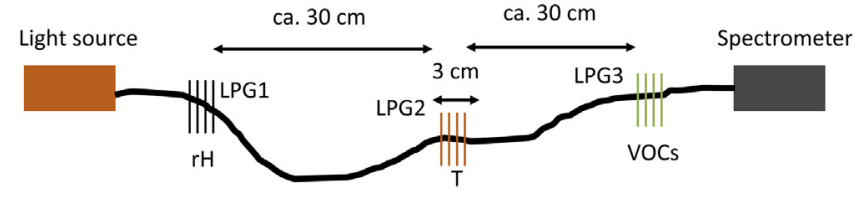

LPG1 $=110.9 \mu \mathrm{m} \quad L P G 2=110 \mu \mathrm{m} \quad L P G 3=110.8 \mu \mathrm{m}$

Fig. 1. Schematic illustration of the LPG sensor array; the individual LPGs were used to measure: LPG1 relative humidity (RH); LPG2 temperature; and LPG3 volatile organic compounds (VOCs).

\section{Materials and methods}

\subsection{Materials}

Poly(allylamine hydrochloride) PAH ( $\mathrm{M}_{\mathrm{w}}$ : 75,000), $\mathrm{NaOH} 1 \mathrm{M}$ aqueous solution, benzene, toluene, chloroform and acetone were purchased from Sigma-Aldrich. $\mathrm{SiO}_{2}$ nanoparticles (NPs) (SNOWTEX 20L) were purchased from Nissan Chemical. Psulphanatocalix[8]arene (CA[8]) $1 \mathrm{mM}$ solution was synthetized in the laboratory [15]. All of the chemicals were analytical grade reagents and used without further purification. Deionized water $(18.3 \mathrm{M} \Omega \mathrm{cm}$ ) was obtained by reverse osmosis followed by ion exchange and filtration (Millipore, Direct-QTM).

\subsection{Fabrication of the array}

LPGs with grating periods of $110.0,110.9$ and $110.8 \mu \mathrm{m}$, all of length $40 \mathrm{~mm}$, were fabricated in boron-germanium co-doped optical fibre (Fibercore PS750) with cut-off wavelength $670 \mathrm{~nm}$ in a point-by-point fashion, side-illuminating the optical fibre by the output from a frequency-quadrupled Nd:YAG laser, operating at $266 \mathrm{~nm}$ [29]. The sensing array was fabricated by splicing together the 3 individual LPGs, with adjacent LPGs separated by a length of $30 \mathrm{~cm}$ of optical fibre with its buffer coating intact, which avoids the creation of in-fibre Mach-Zehnder interferometers [30]. The sensor array is illustrated in Fig. 1. The grating period was selected such that the LPGs operated at or near the phase matching turning point $[15,22]$, which, for coupling to a particular cladding mode (in this case $\mathrm{LP}_{019}$ ), ensured optimized sensitivity.

The transmission spectrum of the optical fibre was recorded by coupling the output from a tungsten-halogen lamp (Ocean Optics HL-2000) into the fibre, analysing the transmitted light using a fibre coupled CCD spectrometer (Ocean Optics HR4000), Fig. 1.

The periods of LPGs were selected in such a way that each of them can be associated with distinct and uniquely identifiable resonance band in the spectrum, which allowed multi-parameter measurements where each LPG was measuring temperature, relative humidity or VOCs (see Section 3 for more details). In particular, LPG1, of period $110.9 \mu \mathrm{m}$, was modified with 5 layers of $\mathrm{SiO}_{2} \mathrm{NPs}$ and was used for RH measurements. LPG2, of period $110.0 \mu \mathrm{m}$ was used for temperature measurement and was uncoated. LPG3, of period $110.8 \mu \mathrm{m}$, was coated with 8 layers of the $\mathrm{SiO}_{2} \mathrm{NPs}$ thin film, which was subsequently infused with calixarene molecules and used for VOCs measurements.

\subsection{Thin film deposition}

Mesoporous thin films of $\mathrm{SiO}_{2}$ NPs were deposited onto LPG1 and LPG3 using an electrostatic self-assembly approach that has been described elsewhere [14,21,25-27]. The region of the optical fibre containing the LPG was fixed in a Teflon holder constructed with a compartment to accommodate a solution [27]. The optical fibre was rinsed with deionized water and immersed in a $1 \mathrm{wt} \%$ $\mathrm{KOH}$ in ethanol/water $=3: 2, \mathrm{v} / \mathrm{v}$ solution for $20 \mathrm{~min}$, leading to a

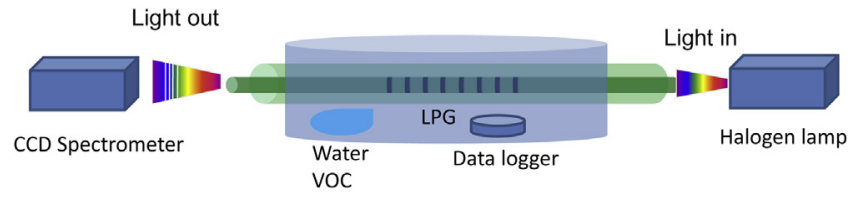

Fig. 2. Schematic illustration of the experimental set-up used for calibrating the response of the single LPG and of the LPG array to relative humidity and to VOCs. The same set-up was used to investigate the exposure of LPG to high concentration of VOCs. In this case, instead of the water, a droplet of the VOC was placed inside the dish.

negatively charged surface. The optical fibre was then immersed sequentially into a solution containing a positively charged polymer, $\mathrm{PAH}$, and a solution containing negatively charged $\mathrm{SiO}_{2} \mathrm{NPs}$, for $20 \mathrm{~min}$ each, resulting in the alternate deposition of PAH and $\mathrm{SiO}_{2}$ NPs layers on the surface of the fibre. The fibre was rinsed with distilled water and dried by flushing with nitrogen gas after each deposition step. A 5 cycle $\left(\mathrm{PAH} / \mathrm{SiO}_{2}\right)_{5}$ film was deposited onto LPG1 and 8 cycle $\left(\mathrm{PAH} / \mathrm{SiO}_{2}\right)_{8}$ was deposited onto LPG3. The number of layers was chosen carefully such that the device possesses sufficient sensitivity to the measurand and that the resonance bands corresponding to the individual LPGs could be resolved after the deposition of the coatings and, in the case of LPG3, after infusion of the functional material.

To sensitise LPG3 to VOCs, the $\left(\mathrm{PAH} / \mathrm{SiO}_{2}\right)_{8}$ coated LPG was immersed into an aqueous solution of $1 \mathrm{mM}$ of $\mathrm{CA}[8]$ for $2 \mathrm{~h}$ and was subsequently washed and dried.

\subsection{Sensor calibration}

The temperature responses of the LPGs were characterised over a range from $10^{\circ} \mathrm{C}$ to $85^{\circ} \mathrm{C}$ by placing the LPGs in an environmental chamber equipped with fibre-optic feed-through. This characterisation process was undertaken for each LPG individually, before splicing and coating, and was repeated after the LPGs had been spliced to form the array and after LPGs 2 and 3 had been coated with the silica nanospheres. The transmission spectra were saved at each temperature, 3 min after thermal equilibrium was reached. A temperature data logger (iButton ${ }^{\circledR}$ Hygrochron Temperature/Humidity Logger, part number DS1923, from Maxim Integrated ${ }^{\mathrm{TM}}$ ), with precision of $\pm 0.5^{\circ} \mathrm{C}$ and $\pm 0.6 \mathrm{RH} \%$, was placed in close proximity to the LPGs. The central wavelengths of the resonance bands were determined from the recorded transmission spectrum using in-house developed software that differentiates the smoothed spectra to identify the zero crossing points.

Similarly to the calibration of the temperature sensitivity of the LPGs, described in Section 3.1, the response to RH was characterised for each LPG individually, before splicing and coating, and was repeated after the LPGs had been spliced to form the array and after LPGs 2 and 3 had been coated with the silica nanospheres.

The LPGs (individual and array) were fixed inside a small cell (Petri dish, volume $100 \mathrm{~cm}^{3}$ ), into which a water droplet was injected, as shown in Fig. 2. The LPGs were maintained straight and taut to avoid bend-induced distortion of the spectrum. The increase of the RH in the cell was monitored using the RH data logger. The transmission spectrum and data logger values were recorded every $10 \mathrm{~s}$.

The performance of LPG3 as a VOC sensor was assessed by exposing it to high concentrations of chloroform, toluene, benzene and acetone vapours. LPG3 was coated with $\left(\mathrm{PAH} / \mathrm{SiO}_{2}\right)_{5}$ and infused with $\mathrm{CA}[8]$ and the central wavelength of the LPG3-R resonance band (identified in the spectrum shown in Fig. 3(a)) was monitored.

The LPGs were fixed in the same cell that was used for RH calibration (as shown in Fig. 2) and a solution of the particular VOC was 
(a)

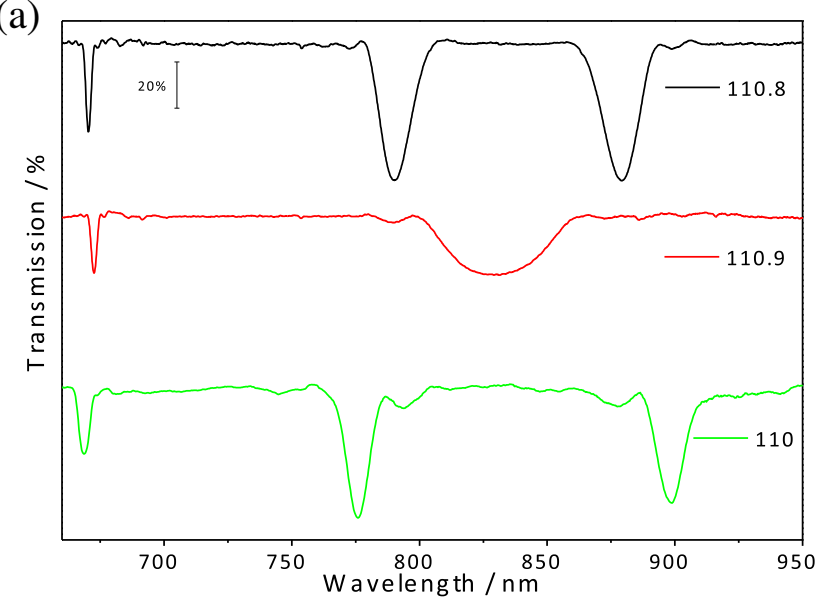

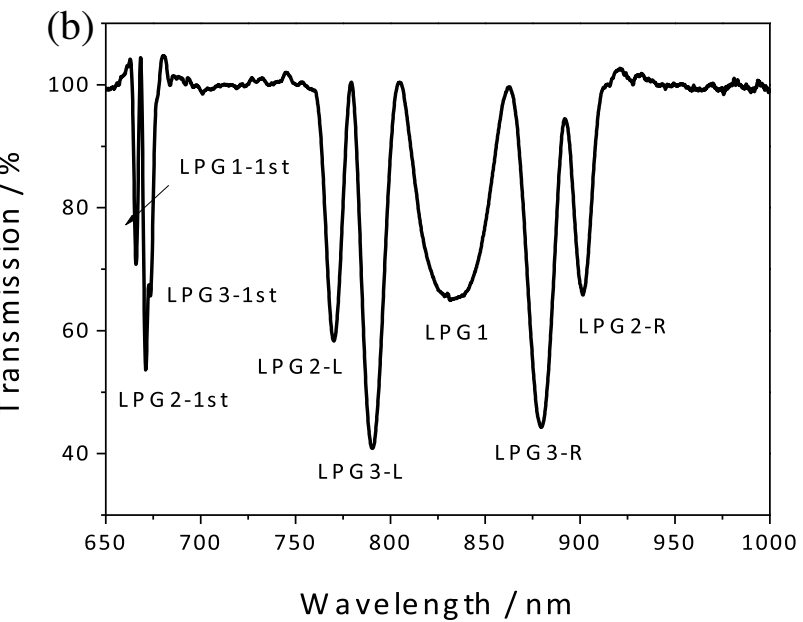

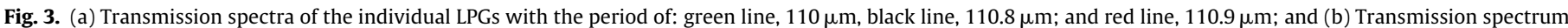

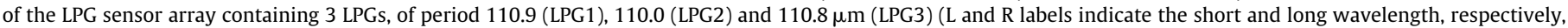

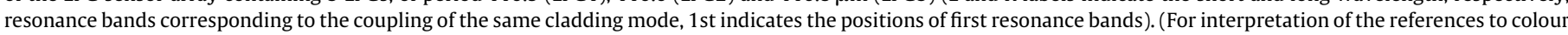
in this figure legend, the reader is referred to the web version of this article.)

injected by syringe using the following volumes: $10 \mu \mathrm{l}$ of toluene, $50 \mu \mathrm{l}$ of benzene and chloroform and $100 \mu \mathrm{l}$ of acetone.

\subsection{Experiments in the real environment}

To investigate the performance of the sensor array in real environments, the system was deployed in two offices and in a laboratory:

1) in an open-plan office area with mechanical ventilation (Vincent Building, Cranfield University) over a $24 \mathrm{~h}$ period (sampling rate $10 \mathrm{~min}$ ), further referred as Vincent Building experiment;

2) in a research laboratory (Whittle building, Cranfield University) over a period of $60 \mathrm{~h}$ (sampling rate $5 \mathrm{~min}$ ), further referred as Whittle Building lab experiment;

3 ) in an office with natural ventilation (Whittle Building, Cranfield University) over a $60 \mathrm{~h}$ period (sampling rate $5 \mathrm{~min}$ ), further referred as Whittle Building office experiment.

Since there is no reliable and sensitive portable sensor for VOCs measurements that can be used for the benchmarking and the VOCs levels in the ambient environment are relatively low and lower that the limit of detection of the LPG VOC sensor, the studies of performance in real environments focussed on measurements of RH and temperature in different building areas, using the temperature and humidity data logger for benchmarking.

\section{Results and discussion}

\subsection{Sensor fabrication}

The transmission spectra of the individual LPGs measured before splicing into the array are shown in Fig. 3a. The periods of the LPGs were chosen such that, when the 3 LPGs were combined, the resonance bands corresponding to the individual LPGs could be resolved. Consequently, the period of LPG1 is such that it couples light from the core to the $\left(\mathrm{LP}_{019}\right)$ mode near the phase matching turning point, just before band splits (Fig. 3a, red line). Decrease of the grating period leads to the splitting of the resonance band (these bands were labelled LPG1-L and LPG1-R, indicating short and long wavelength), as shown in Figs. 3a, S1a and b [29]. The transmission spectrum of the LPG array after splicing, but before LPG1 and LPG3 were coated, is shown in Fig. 3b, where distinct resonance bands corresponding to each of the LPGs are observed clearly. The "U" shape band labelled LPG1 is associated with the bare LPG operating at the PMTP. This band further splits into two, hereafter referred to as LPG2-L and LPG2-R, after the deposition of the sensitive coating.

\subsection{Functional coating deposition}

Deposition of the $\left(\mathrm{PAH} / \mathrm{SiO}_{2}\right)_{5}$ film leads to the changes in the transmission spectrum of LPG1 (Fig. S2), which agree with previous observations [21]. In particular, the deposition of the first layer was observed to cause the resonance band of LPG1 to split into two (labelled LPG1-L and LPG1-R). Further increase of the film thickness resulted in changes of the central wavelengths of the resonance bands until they merged with the resonance bands of LPG2 and LPG3.

The evolution of the transmission spectrum during the deposition of the $\left(\mathrm{PAH} / \mathrm{SiO}_{2}\right)_{8}$ film onto LPG3 is shown in Fig. S3. A continuous linear shift of resonance bands' central wavelengths was observed when LPG3 was immersed into the $\mathrm{SiO}_{2}$ solution decrease for LPG3-L, increase for LPG3-R, Fig. S3b. The resonance bands corresponding to LPGs 2 and 3 merged after deposition of the 2nd layer (labelled as LPG2+3-R). Due to the overlap of the resonance bands of LPG1 and LPG3, it was difficult to observe their wavelength shift when LPG transmission spectra were measured in air, Fig. S3a. It should be noted that overlap of the resonance bands could limit sensor performance, introducing cross-talk between sensors and ambiguity if the peaks merge or cross-over. The modification of the senor surface was conducted in such a way that this cross-talk was avoided and bands were clearly distinguishable. The thickness of the coating was also chosen to provide optimal sensitivity to the appropriate measureands and to bias the central wavelengths of the attenuations bands of the individual LPGs such that they could be resolved when the LPGs were arranged to be in-series in a single optical fibre.

The central wavelengths of the dual resonance bands of LPG3 were observed to change as the $\mathrm{CA}[8]$ infused into the coating and changed the coating refractive index, as shown in Fig. 4a. The evolution of the resonance bands is shown in Figs. 4b and S4. This shift of the resonance bands in response the change in the coating's RI enables the discrimination of the LPG2-R and LPG3-R when the LPGs were in air. The corresponding changes of the transmission spectrum are shown in Fig. 4c. The process was terminated after no further shift in the attenuation bands was observed, which took 
(a)

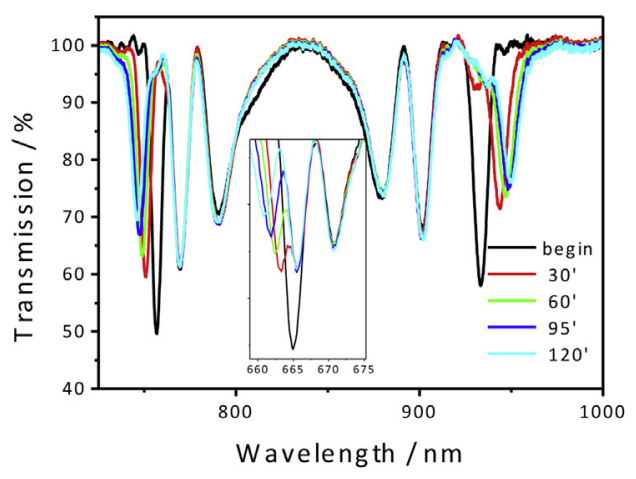

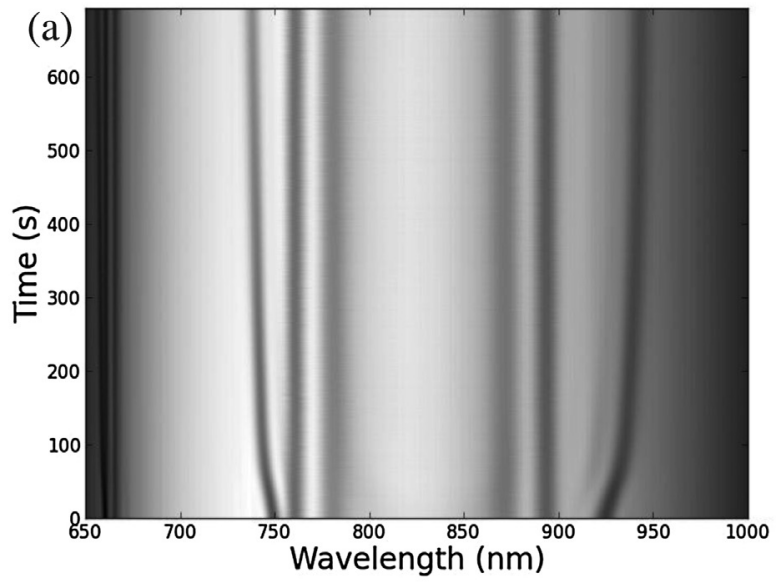

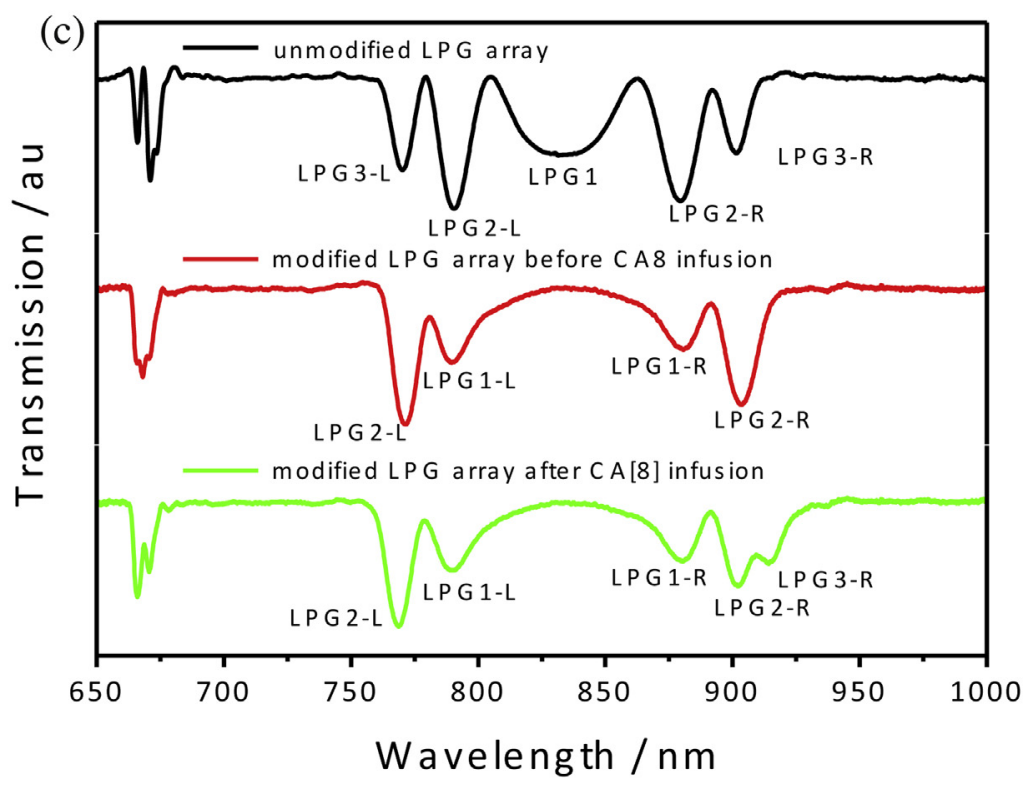

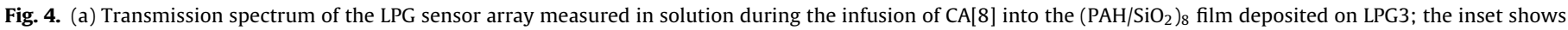

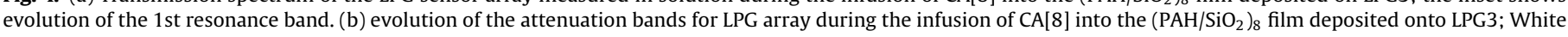

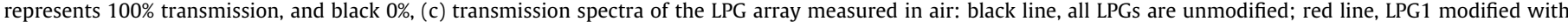

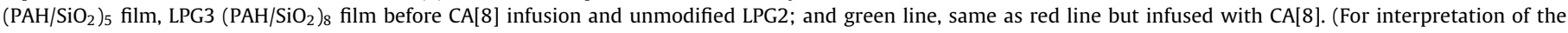
references to colour in this figure legend, the reader is referred to the web version of this article.)

approximately two hours. It should be also noted that the resonance bands associated with LPG1 and LPG2 remained unchanged.

Fig. 4c compares the transmission spectrum of the sensor array before and after deposition of the $\left(\mathrm{PAH} / \mathrm{SiO}_{2}\right)_{8}$ film and after infusion of $\mathrm{CA}[8]$ into the $\left(\mathrm{PAH} / \mathrm{SiO}_{2}\right)_{8}$ film. The transmission spectrum of the modified sensor array shows distinct resonance bands, allowing the multiplexing of the sensors in the wavelength domain.

\subsection{Temperature calibration}

Fig. S5 shows the shift of the central wavelength of the single unmodified LPG3 (before splicing) induced by the temperature change. Figs. 5 and S6 show transmission spectra of all 3 spliced LPGs recorded at different temperatures. The resonance bands of all of the LPGs can be resolved throughout the measurement range. Fig. 5b shows the calibration plot of the central wavelength vs tem- perature for the longer wavelength (labelled $\mathrm{R}$ ) resonance bands of LPG1, LPG2 and LPG3.

The temperature response of LPG2-R, recorded during three calibration tests, is shown in Fig. S7a. The small offsets in the central wavelength recorded at the same temperature during the three experiments may be associated with a difference in the strain applied to the fibre when it was mounted, which was to ensure that the fibre was not bent. It is known that an LPG sensor operating at the phase matching turning point is extremely sensitive to temperature, strain, surrounding refractive index and bending $[15,22]$. To take this into account, the temperature response was measured several times and the weighted average value of the calibration curve was used when determining the temperature during the subsequent experiments undertaken in real environments.

The temperature response was also measured after deposition of the $\mathrm{PAH} / \mathrm{SiO}_{2}$ film onto LPG3. The presence of the coating reduced the temperature sensitivity of the LPG3-R resonance band from 
(a)

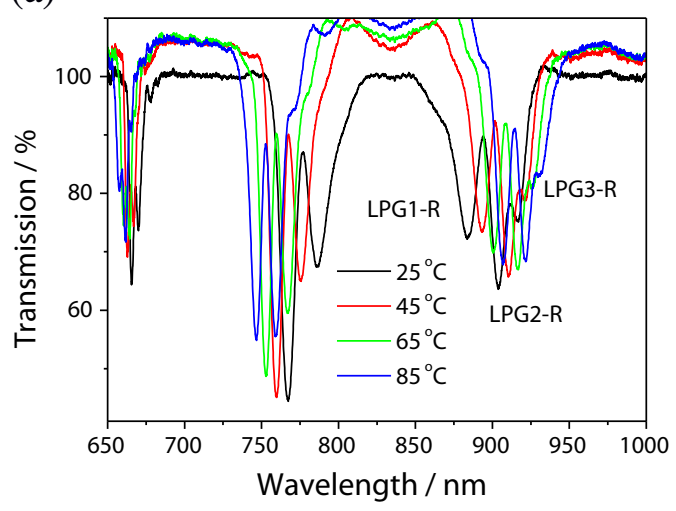

(b)

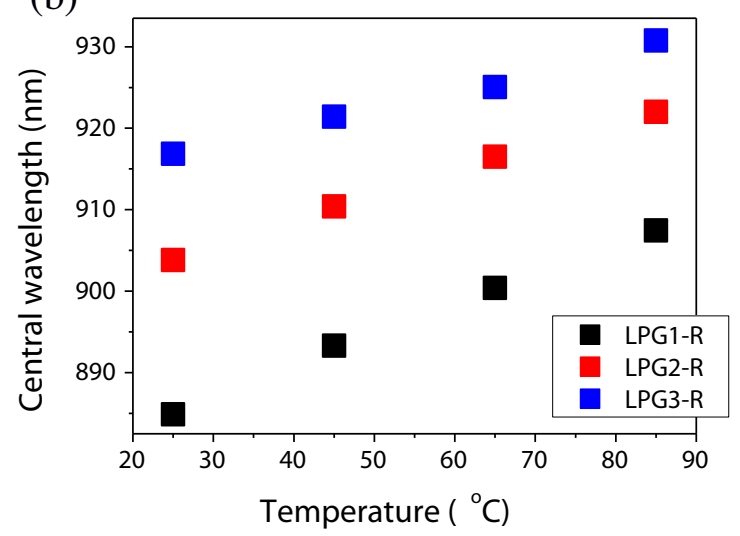

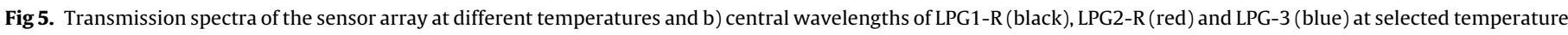

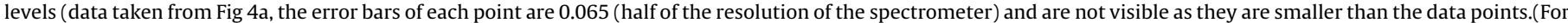
interpretation of the references to colour in this figure legend, the reader is referred to the web version of this article.)

$0.45 \pm 0.02$ to $0.25 \pm 0.01 \mathrm{~nm}^{\circ} \mathrm{C}^{-1}$, Fig. S7b. There are two factors that can change the sensor's response to temperature after modification with $\mathrm{PAH} / \mathrm{SiO}_{2}$ film: a change in the $\mathrm{RH}$ with temperature (since the mesoporous film is sensitive to $\mathrm{RH}$ ) and the thermooptical properties of the $\mathrm{PAH} / \mathrm{SiO}_{2}$ film (a change of the RI of the film with temperature). Relative humidity decreases with increasing temperature is suggested to be the main cause of the reduced gradient (Fig. S8).

In order to obtain the real temperature sensitivity of the LPG modified with $\mathrm{PAH} / \mathrm{SiO}_{2}$ film and to take into account the effect of the $\mathrm{RH}$, the gradient of the temperature response was recalculated using the following Eq. (2):

$\Delta \lambda_{\text {total }}=\Delta \lambda_{\mathrm{T}}+\Delta \lambda_{\mathrm{RH}}$

where $\Delta \lambda_{\text {total }}$ is the measured total temperature induced wavelength shift of the resonance band of the LPG modified with the $\mathrm{PAH} / \mathrm{SiO}_{2}$ film, $\Delta \lambda_{\mathrm{T}}$ is the wavelength shift caused by temperature only (this was plotted as a calibration curve, Fig. S8); and $\Delta \lambda_{\mathrm{RH}}$ is the wavelength shift caused by $\mathrm{RH}$ only (determined from the $\mathrm{RH}$ calibration curve of the LPG modified with the $\mathrm{PAH} / \mathrm{SiO}_{2}$ film).

This process revealed that the temperature sensitivities of the coated LPG were similar to that measured before the coating deposition (Fig. S8). This recalculation was also used when processing the data from the measurements undertaken in laboratory and office environments, which will be described in Section 4.

The temperature sensitivities of resonance bands LPG1-R, LPG2$\mathrm{R}$ and LPG3-R (after all modifications) were determined to be $0.40 \pm 0.02 \mathrm{~nm}^{\circ} \mathrm{C}^{-1}, 0.45 \pm 0.02 \mathrm{~nm}^{\circ} \mathrm{C}^{-1}$ and $0.25 \pm 0.01 \mathrm{~nm}^{\circ} \mathrm{C}^{-1}$ respectively and are summarized in Table 1 . The detection limit of the LPG array was $0.29 \pm 0.01{ }^{\circ} \mathrm{C}$, determined from the LPG2-R calibration curve (Fig. S7a), taking into account the spectral resolution of the spectrometer used $(0.13 \mathrm{~nm})$. It should be noted that the differences between the slopes for individual LPGs arises from the different LPG period and the thickness and type of the coating (discussed earlier).

Table 1

Comparison of the sensitivities of the resonance bands of the LPGs to temperature.

\begin{tabular}{ll}
\hline LPG & Sensitivity $\left[\mathrm{nm}^{\circ} \mathrm{C}^{-1}\right]$ \\
\hline LPG1-L modified with $\left(\mathrm{PAH} / \mathrm{SiO}_{2}\right)_{5}$ & $0.40 \pm 0.02^{\mathrm{a}}$ \\
LPG1-R modified with $\left(\mathrm{PAH} / \mathrm{SiO}_{2}\right)_{5}$ & $0.45 \pm 0.02^{\mathrm{a}}$ \\
LPG2-L unmodified & $-0.50 \pm 0.02^{\mathrm{a}}$ \\
LPG2-R-unmodified & $0.45 \pm 0.02^{\mathrm{a}}$ \\
LPG3-R modified with $\left(\mathrm{PAH} / \mathrm{SiO}_{2}\right)_{8}+$ infused with CA[8] & $0.25 \pm 0.01^{\mathrm{a}}$ \\
\hline
\end{tabular}

a The error indicates the standard deviation of 3 separate experiments.

\subsection{RH calibration}

No change in the transmission spectrum of LPG2-R (without coating) was observed when the array was exposed to $\mathrm{RH}$ changes in the range $40-70 \%$. Single, unmodified LPG1 and LPG3 were examined in the same way and it has been shown that, within the measurement resolution, uncoated LPGs are not sensitive to $\mathrm{RH}$, Fig. S9. The sensitivity of the modified LPG sensors arises from the changes in the RI of the mesoporous coating when water molecules infuse into the coating. Interestingly, when LPG3, modified with $\left(\mathrm{PAH} / \mathrm{SiO}_{2}\right)_{8}$ and infused with $\mathrm{CA}[8]$, was exposed to humidity, the sensitivity was much smaller than that of LPG1 with the $\left(\mathrm{PAH} / \mathrm{SiO}_{2}\right)_{5}$ coating but without $\mathrm{CA}[8]$. Most plausibly this is a result of the hydrophobicity of the $\mathrm{CA}[8]$, which, when infused into the $\mathrm{PAH} / \mathrm{SiO}_{2}$ film, suppress water adsorption.

$\mathrm{RH}$ calibration curves of 3 separate measurements conducted for LPG1-R modified with the $\left(\mathrm{PAH} / \mathrm{SiO}_{2}\right)$ film are shown in Fig. $6 \mathrm{a}$. Similar to the temperature measurements, the starting central wavelength of the resonance band was different (explained in the Section 3.1). Differences in the ambient temperature will also influence the starting central wavelength as well. The average slope was used to determine the values of $\mathrm{RH}$ during experiments undertaken in the laboratories and offices. The detection limit of the LPG array was $1.39 \mathrm{RH} \%$, determined from weighted average of the slopes $(0.09 \pm 0.01 \mathrm{~nm}$ per $1 \% \mathrm{RH}$ change $)$ obtained from the calibration curves (Fig. 6a) and spectral resolution of the spectrometer $(0.13 \mathrm{~nm})$. The shift of the central wavelengths in response to a change in RH for LPG1-L and LPG1-R is shown in Figs. S10a and 6b respectively. By monitoring the transmission at a fixed wavelength, selected to midway up the slope of the resonance band of LPG1-R, the response to the RH change can also be observed, Fig. S10b.

\subsection{VOCs calibration}

No changes in the central wavelengths of the resonance bands corresponding to LPG1, modified with the $\left(\mathrm{PAH} / \mathrm{SiO}_{2}\right)_{5}$ film, and unmodified LPG2 were observed when the sensor array was exposed to chloroform, as can been seen in Fig. 7a. A small, but measurable shift of the resonance band corresponding to LPG3, modified with the $\left(\mathrm{PAH} / \mathrm{SiO}_{2}\right)_{8}$ infused with $\mathrm{CA}[8]$, was observed at high VOCs concentrations, Fig. 7b, S11 and Table 2.

The low sensitivity to VOCs was, most plausibly, a results of non-optimal grating period and film thickness. In this case, we have demonstrated the potential for the use of the LPG for VOCs detection at high concentration as part of the sensing array. Future 
(a)

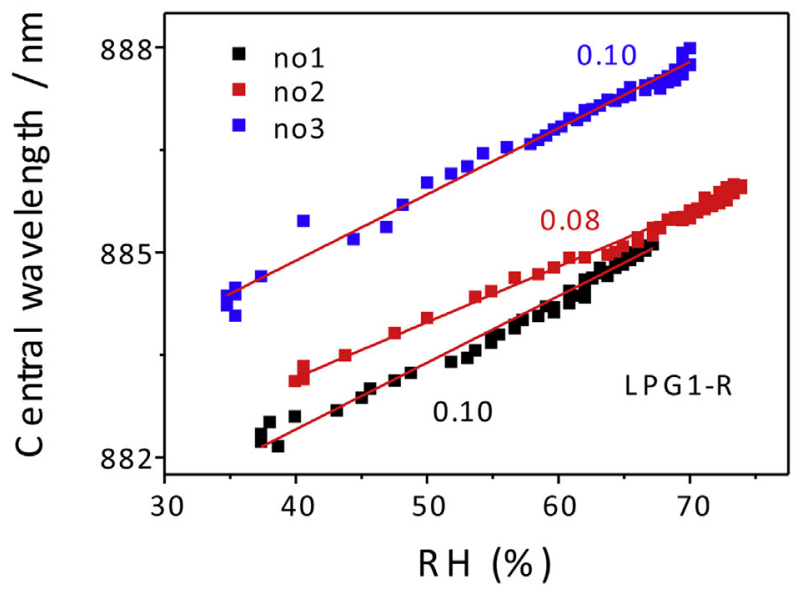

(b)

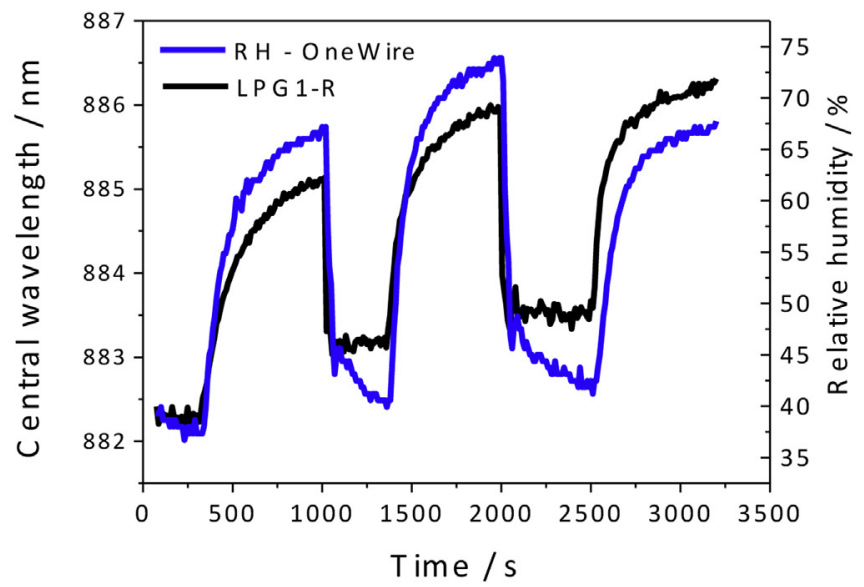

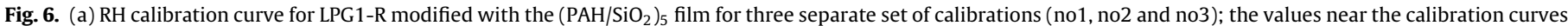

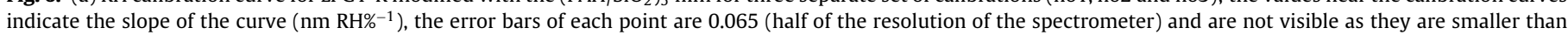

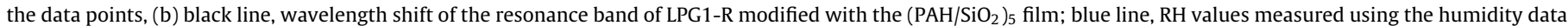
logger. (For interpretation of the references to colour in this figure legend, the reader is referred to the web version of this article.)
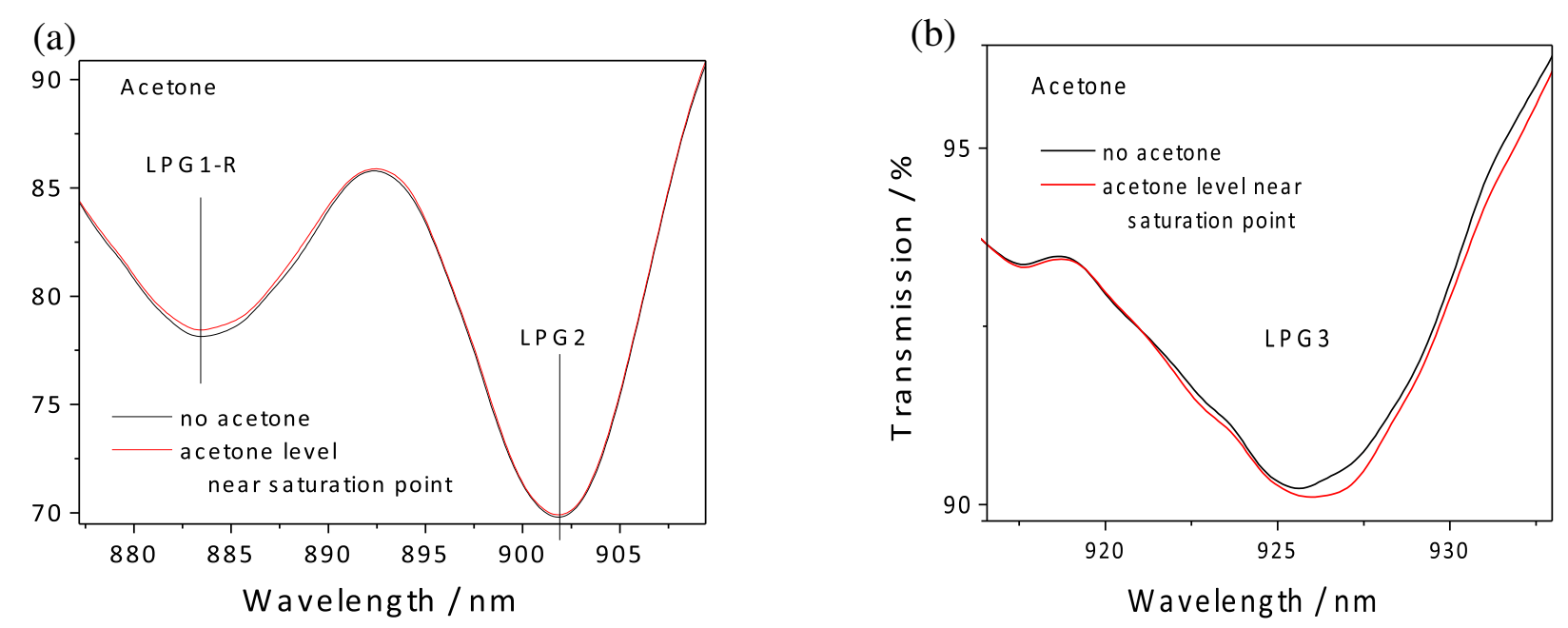

Fig. 7. Changes in the transmission spectra of the LPG array, (a) LPG1-R, LPG2 and (b) LPG3, in response to exposure to a high concentration of acetone.

Table 2

LPG3 performance during VOCs experiments.

\begin{tabular}{llll}
\hline VOC & $\begin{array}{l}\text { Amount of } \\
\text { solution }\end{array}$ & $\begin{array}{l}\text { Estimated } \\
\text { concentration }\end{array}$ & $\begin{array}{l}\text { Central } \\
\text { wavelength } \\
\text { shift }\end{array}$ \\
\hline Acetone & $100 \mu \mathrm{l}$ & $298,000 \mathrm{ppmv}$ & $0.35 \pm 0.065 \mathrm{~nm}^{\mathrm{a}}$ \\
Benzene & $50 \mu \mathrm{l}$ & $125,000 \mathrm{ppmv}$ & $0.23 \pm 0.065 \mathrm{~nm}^{\mathrm{a}}$ \\
Chloroform & $50 \mu \mathrm{l}$ & $210,000 \mathrm{ppmv}$ & $0.58 \pm 0.065 \mathrm{~nm}^{\mathrm{a}}$ \\
Toluene & $10 \mu \mathrm{l}$ & $28,000 \mathrm{ppmv}$ & $0.24 \pm 0.065 \mathrm{~nm}^{\mathrm{a}}$ \\
\hline
\end{tabular}

a Error bars represent the half of the resolution of the spectrometer.

work will focus on studying this issue in more detail. The factors to consider that can lead to the improved sensitivity include the deposition of the functional film with different thicknesses, optimising grating period, as well as use of different types of calixarene molecules as different geometries with different cavities may lead to improved interaction of VOCs with the coating.

\subsection{Experiments undertaken in real environments}

During the experiment in the Vincent Building, the temperature and $\mathrm{RH}$ values ranged from 23.1 to $24.6^{\circ} \mathrm{C}$ and 32.1 to $53.7 \%$, respectively. The unmodified LPG2 and LPG1 modified with the $\left(\mathrm{PAH} / \mathrm{SiO}_{2}\right)_{5}$ film were used to measure temperature and $\mathrm{RH}$. Using the calibration curves described in Sections 3.1 and 3.2, the $\mathrm{RH}$ and temperature measured using the sensor array and data logger were compared, as shown in Fig. 8a and b, respectively.

Although extra care had been taken to anchor the LPGs firmly to keep them taut and straight during the measurements, it was found that they relaxed over the extended measurement period $(>24 \mathrm{~h})$. This relaxation caused a slight shift of the central wavelength with elapsed time. To take wavelength shift caused by bending as the result of LPG relaxation into account a correction coefficient (time slip) was introduced that was found to be $0.0067 \mathrm{~nm} \mathrm{~h}^{-1}$ and was applied to the evaluation of temperature during the Whittle Building office experiment and RH during experiment in Vincent Building, Figs. 8a and S14c . The effect of time slip coefficient was negligible in all other cases due to the small range of the measured values. This effect was not observed during the calibration experiments, as their duration was typically $4 \mathrm{~h}$.

The Fig. 8a shows the effect of the time slip coefficient onto $\mathrm{RH}$ evaluation. It can be seen that for the first $15 \mathrm{~h}$ there is no significant difference between RH measured using LPG sensor and commercial humidity sensor. After $15 \mathrm{~h}$, however, the small deviation in the $\mathrm{RH}$ 
(a)

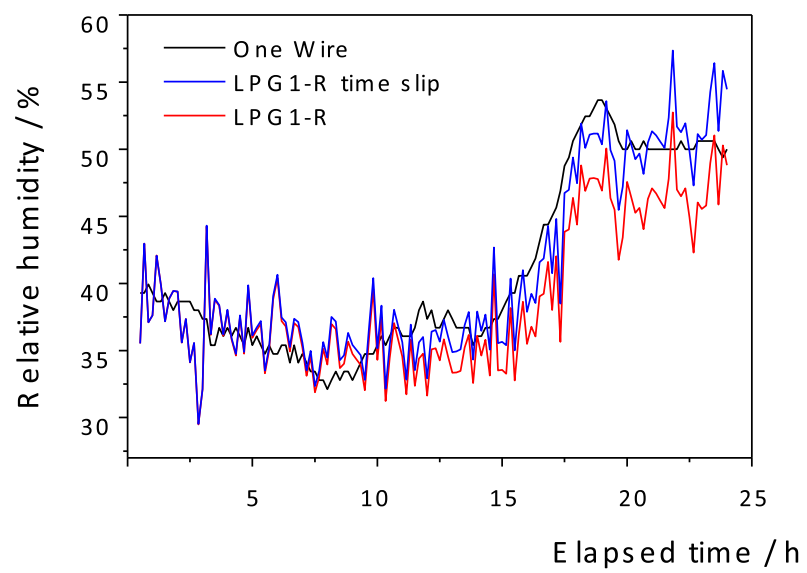

(b)

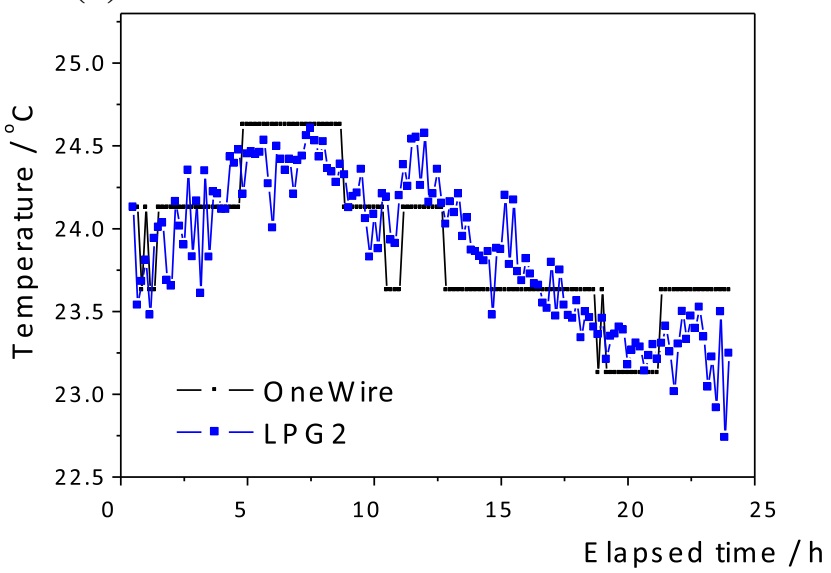

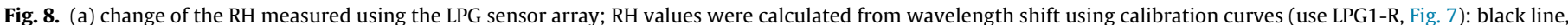

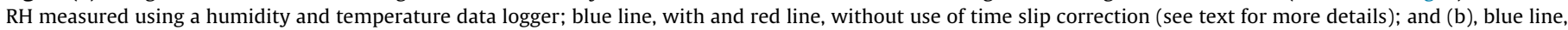

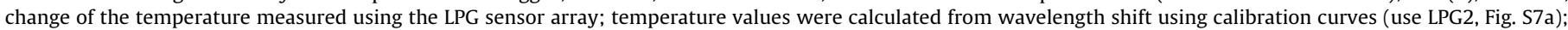

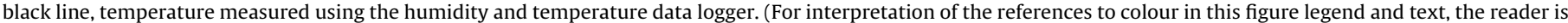
referred to the web version of this article.)

values between LPG sensors and humidity logger was observed, red line Fig. 8a. Very good correlation between LPG and commercial sensor was observed when the time slip coefficient was introduced (blue line Fig. 8a).

To investigate the correlation between the measurements performed by the data logger and the LPG sensor array, the temperature and RH values measured using the data logger were plotted against the LPG sensor array (Fig. S12). The slope of the linear fit within these values was higher than 0.99 which indicates strong correlation between the sensing array and the data logger (Fig. S12).

The data recorded by the system during the Whittle building lab and office experiments are shown in Figs. S13 and S14, respectively. In the Whittle building lab experiment, the temperature and $\mathrm{RH}$ values ranged from 21.6 to $24.1^{\circ} \mathrm{C}$ and 42.5 to $61.4 \%$ respectively and the data measured by the LPG sensor array and the data logger were in a good agreement, Fig. S14. The values for temperature and $\mathrm{RH}$ ranged from 22.1 to $28.6^{\circ} \mathrm{C}$ and 34.8 to $57.3 \%$ respectively in Whittle building office area, Fig. S14. The time slip correction was used for temperature values calculation in this case, Fig. S14.

The average difference between the values measured by data logger and the values calculated based on performance of the array was below $0.5{ }^{\circ} \mathrm{C}$ and $5 \%$ of $\mathrm{RH}$. The achieved accuracy fulfils the guidelines and recommendations for temperature and $\mathrm{RH}$ indoor given in the units of ${ }^{\circ} \mathrm{C}$ [31] or tens of RH percent respectively [7].

As expected, the concentration of VOCs in real environment was lower that the detection limit of the LPG, such that the resonance bands of LPG3 showed no response. The concentration of VOCs in real environment can increase significantly when new carpets are installed or after painting the walls. We have previously demonstrated that an LPG modified with a $\left(\mathrm{PAH} / \mathrm{SiO}_{2}\right)_{8}$ coating infused with $\mathrm{CA}[8]$ can be used successfully to measure VOCs emitted from the paint [32].

Although there is a range of sensors developed for IAQ monitoring, the fibre optic sensor offers the ability to perform multi-parameter and distributed measurements of the IAQ monitoring that potentially can allow increasing the efficiency of the building energy performance. We have demonstrated the feasibility of measuring three different parameters. Measurement of other IAQ factors such as carbon monoxide or dioxide together with temperature and $\mathrm{RH}$ are possible using the same principle. Other advantages of the fibre optic sensors include multipoint sensing with a single interrogation set-up.
One of the challenges in multiplexing an array of the LPGs is signal processing step that allows clear identification of the attenuation bands corresponding to the each of the individual LPG sensor. The central wavelengths of the attenuation bands were determined in real time using the peak detection routine in LabVIEW to process the spectra recorded by the Ocean Optics spectrometer. The central wavelengths of the resonance bands were determined by differentiation of the smoothed spectra to identify the zero crossing points.

This can be further improved by other signal processing techniques described in [30] and [33] allowing clearly recognising the central wavelengths change according to the changes of the measurands in the surrounding environment.

\section{Conclusions}

An optical fibre LPG sensor array was developed successfully and its performance assessed with the aim of simultaneous multiparameters measurements of temperature and relative humidity, key IAQ parameters. It was demonstrated that performance of sensor array consisting of 3 LPGs, appropriately modified to measure temperature, $\mathrm{RH}$ and VOC concentration, was comparable to commercially available temperature and RH sensors. The sensor array was successfully tested in real environment with difference less than $0.5^{\circ} \mathrm{C}$ and $5 \mathrm{RH} \%$ in comparison with the commercially used sensor.

The future work will focus on optimization of the sensor parameters for VOCs detection.

\section{Acknowledgement}

The authors would lie to acknowledge the support of a UK Engineering and Physical Sciences Research Council (EPSRC) Platform Grant (EP/H02252X/1) and grants EP/L010437 and EP/N025725/1. The underlying data can be accessed at http://dx.doi.org/10.17862/ cranfield.rd.4288373.

\section{Appendix A. Supplementary data}

Supplementary data associated with this article can be found, in the online version, at http://dx.doi.org/10.1016/j.snb.2016.12.050. 


\section{References}

[1] D. Ekmekcioglu, S. Keskin, Charakterization of indoor air particulate matter in selected elementary schools in Istanbul, Turkey, Indoor Built Environ. 16 (2) (2007) 169-176

[2] J. Leech, W.C. Nelson, R.T. Burnett, S. Aaron, M.E. Raizenne, It is about time:A comparison of Canadian and American time-activity patterns, J. Expo. Sci. Environ. Epidemiol. 12 (6) (2002) 427-432.

[3] D. Crump, Investigating indoor air quality problems: best practice and case studies, Proc. Inst. Civil Eng: Forensic Eng. 166 (2) (2013) 94-103.

[4] European Commission Scientific Committee on Health and Environmental Risks (SCHER). Opinion on risk assessment on indoor air quality. s.l., (European Comission, 2007). http://ec.europa.eu/health/ph_risk/committees/ 04_scher/docs/scher_o_055.pdf.

[5] J. Rudge, R. Gilchrist, Excess winter morbidity among older people at risk of cold homes: a population-based study in a London borough, J. Public Health 27 (4) (2005) 353-358.

[6] Heatwave Plan for England, (Public Health England, 2013), https://www.gov. uk/government/uploads/system/uploads/attachment_data/file/201150/ Heatwave_plan_2013_-_Making_the_case_Accessible_updated.pdf.

[7] A.V. Arundel, E.M. Sterling, J.H. Biggin, T.D. Sterling, Indirect health effects of relative humidity in indoor environments, Environ. Health Perspect. 65 (1986) 351-353.

[8] T. Husman, Health effects of indoor-air microorganisms, Scand. J. Work Environ. Health 22 (1) (1996) 5-13.

[9] K.J. Chua, S.K. Chou, W.M. Yang, J. Yan, Achieving better energy-efficient air conditioning-a review of technologies and strategies, Appl. Energy 104 (2013) 87-104

[10] Indoor air quality in office buildings: A technical guide, (Government of Canada Publications, 1995). http://publications.gc.ca/collections/Collection/ H46-2-93-166Erev.pdf.

[11] C. Yu, D. Crump, A review of the emission of VOCs from polymeric materials used in buildings, Build. Environ. 33 (6) (1998) 357-374.

[12] A.D. Kersey, A review of recent developments in fiber optic sensor technology, Opt. Fiber Technol. 2 (3) (1996) 291-317.

[13] T. Venugopalan, T. Sun, K.T.V. Grattan, Long period grating-based humidity sensor for potential structural health monitoring, Sens. Actuators A 148 (1) (2008) 57-62.

[14] S. Korposh, R. Selyanchyn, W. Yasukochi, S.-W. Lee, S.W. James, R.P. Tatam, Optical fibre long period grating with a nanoporous coating formed from silica nanoparticles for ammonia sensing in water, Mater. Chem. Phys. 173 (2-3) (2012) 784-792

[15] S.M. Topliss, S.W. James, F. Davis, S.P.J. Higson, R.P. Tatam, Optical fibre long period grating based selective vapour sensing of volatile organic compounds, Sens. Actuators B 143 (2) (2010) 629-634.

[16] F.J. Arregui, I.R. Matias, J.M. Corres, I. Del Villar, J. Goicoechea, C.R. Zamarrenoa, M. Hernáez, R.O. Claus, Optical fiber sensors based on layer-by-layer nanostructured films, Proc. Eng. 5 (2010) 1087-1090.

[17] C. Elosua, C. Bariain, I.R. Matias, Optical fiber sensors to detect volatile organic compound in sick building syndrome applications, Open Constr. Build. Technol. J. 4 (1) (2005) 113-120.

[18] G. Rego, A review of refractometric sensors based on long period fibre gratings, Sci. World J. 2013 (2013) 1-14.

[19] S. James, R. Tatam, Optical fibre long-period grating sensors: characteristics and application, Meas. Sci. Technol. 14 (5) (2003) R49-R61.

[20] V. Bhatia, D.K. Campbell, D. Sherr, T.G. D’Alberto, N.A. Zabaronick, G.A. Ten Eyck, K.A. Murphy, R.O. Claus, Temperature-insensitive and strain-insensitive long-period grating sensors for smart structures, Opt. Eng. 36 (7) (1997) 1872-1875.

[21] S. Korposh, S.-W. Lee, S.W. James, R.P. Tatam, Refractive index sensitivity of fibre-optic long period gratings coated with $\mathrm{SiO}_{2}$ nanoparticle mesoporous thin films, Meas. Sci. Technol. 22 (7) (2011) 075208.

[22] X. Shu, L. Zhang, I. Bennion, Sensitivity characteristics of long-period fiber gratings, J. Lightwave Technol. 20 (2) (2002) 255-266.

[23] A. Cusano, P. Pilla, L. Contessa, A. Iadicicco, S. Campopiano, A. Cutolo, M. Giordano, G. Guerra, High-sensitivity optical chemosensor based on coated long-period gratings for sub-ppm chemical detection in water, Appl Phys. Lett. 87 (23) (2005) 1-3.

[24] S.W. James, I. Ishaq, G.J. Ashwell, R.P. Tatam, Cascaded long-period gratings with nanostructured coatings, Opt. Lett. 30 (17) (2005) 2197-2199.

[25] S. Korposh, F. Davis, S.W. James, T. Wang, S.-W. Lee, S. Higson, R.P. Tatam, Detection of volatile organic compounds (VOCs) using an optical fibre long period grating with a calixarene anchored mesoporous thin film, Proc. SPIE 8794 (2016) 87941I.

[26] S. Korposh, T. Wang, S. James, R. Tatam, S.-W. Lee, Pronounced aromatic carboxylic acid detection using a layer-by-layer mesoporous coating on optical fibre long period grating, Sens. Actuators B 173 (2012) 300-309.
[27] S. Korposh, S. Kodaira, S.-W. Lee, J. Batty, S.W. James, Nano-assembled thin film gas sensor II. An intrinsic high sensitive fibre optic sensor for ammonia detection, Sens. Mater. 21 (4) (2009) 179-189.

[28] A.K. Hassan, A.V. Nabok, A.K. Ray, A. Lucke, K. Smith, C.J.M. Stirling, F. Davis, Thin films of calix-4-resorcinarene deposited by spin coating and Langmuir-Blodgett techniques: determination of film parameters by surface plasmon resonance, Mater. Sci. Eng. C 8-9 (1999) 251-255.

[29] R.Y.N. Wong, E. Chehura, S.E. Staines, S.W. James, R.P. Tatam, Fabrication of fiber optic long period gratings operating at the phase matching turning point using an ultraviolet laser, Appl. Opt. 53 (21) (2014) 4669-4674.

[30] R.P. Murphy, S.W. James, R.P. Tatam, Multiplexing of fiber-optic long-period grating-based interferometric sensors, J. Lightwave Technol. 25 (3) (2007) 825-829.

[31] Workplace (Health, Safety and Welfare) Regulations (Health and safety executive, 1992), http://www.hse.gov.uk/pubns/priced/124.pdf.

[32] J. Hromadka, S. James, F. Davis, R.P. Tatam, D. Crump, S. Korposh, Detection of the volatile organic compounds emitted from paints using optical fibre long period grating modified with the mesoporous nano-scale coating, Proc. SPIE 9634, 24th International Conference on Optical Fibre Sensors, 963435 (September 28, 2015), doi: 10.1117/12.2195035.

[33] S.W. James, S. Korposh, S.-W. Lee, R.P. Tatam, A long period grating-based chemical sensor insensitive to the influence of interfering parameters, Opt. Express 22 (7) (2014) 8012-8023.

\section{Biographies}

Jiri Hromadka received both his bachelor and master degrees in 2011 and 2013 respectively in environmental sciences from Charles University in Prague (Czech Republic) and an MSc in Health and the Environment from Cranfield University in 2013. He joined the University of Nottingham in 2013 to start his PhD study with a focus on a development of fibre optic chemical sensors with a special interest in environmental applications.

Dr Serhiy Korposh received both his bachelor and master degrees in 2001 and 2002 respectively in physics from Uzhgorod National University, Transcarpathia (Ukraine) and Ph.D. degree from Cranfield University in 2007. He worked as a postdoctoral researcher on development of the novel materials for chemical sensors in the Graduate School of Environmental Engineering of the University of Kitakyushu from 2008 to 2012. From 2012-2013 he worked as a research fellow in the Department of Engineering Photonics, Cranfield University. He is currently Lecturer at the University of Nottingham. His research interest lies in the field of application and development of fibre-optic chemical sensors modified with the sensitive materials.

Prof Stephen James was born and bred in Llanelli, Wales. He gained an MSc in 1988 in Applied Optics from Imperial College, London, and a PhD from the University of Southampton in 1992. He joined Cranfield University as a research fellow in 1993, where he developed his interest in the development and application of optical instrumentation and sensors. As a Reader, he leads the optical fibre sensing activity in the Department of Engineering Photonics, with a focus on sensing chemical and physical parameters, and on deploying instrumentation in real world environments, ranging from foundation piles, railways, and aerospace structures to superconducting magnets.

Professor Ralph P. Tatam BSc (Hons), PhD, DSc, CSci, CEng, CPhys, FinstP, FSPIEwas appointed to a personal Chair in Engineering Photonics at Cranfield University in 1998. He graduated from Exeter University in 1981 (BSc Physics and Chemistry) and was awarded a PhD in Physics (1986) for work on dynamic Rayleigh-Brillouin laser light scattering studies of liquids. He worked as a Research Fellow and Lecturer in the Physics Laboratory at the University of Kent developing optical fibre sensors from 1985 to 1989. In 1989 he took up an appointment at Cranfield where he has established a research team investigating optical instrumentation and sensors applied to challenging measurement problems in engineering and more recently biomedical. He served as Dean of the Faculty of Engineering and Aerospace for 31/2 years from 2008. He is the European Editor for the journal Measurement Science and Technology and served as the Associate Editor for the SPIE journal Optical Engineering for 5 years and is on the editorial board of several other international journals. Ralph has been on the Technical Programme committee for numerous international conferences, including Symposium Chair for the SPIE Optical Metrology symposium, Munich in 2005 and 2007. He has published over 400 papers in the field of optical instrumentation and sensors, and won the UK National Measurement Awards Frontier Science and Measurement category in 2001 and 2004 for his work on planar Doppler velocimetry and nanostructured films on optical fibres, respectively. In 2005 he was awarded a DSc by Exeter University and elected as a Fellow of the SPIE. 
Multi-parameter measurements using optical fibre long period gratings for indoor air quality monitoring

Hromadka, J.

Elsevier

Hromadka J, Korposh S, Partridge MC et al., Multi-parameter measurements using optical fibre long period gratings for indoor air quality monitoring. Sensors and Actuators B: Chemical, pÿVolume 244, June 2017, Pages 217225.

http://dx.doi.org/10.1016/j.snb.2016.12.050

Downloaded from Cranfield Library Services E-Repository 